PATIENT-RELATED OUTCOME MEASUREMENTS IN PLEURAL EFFUSIONS

I Psallidas, JP Corcoran, EK Mishra, RJ Hallifax, NM Rahman. Oxford Centre for Respiratory Medicine Oxford University Hospitals NHS Trust, Oxford, UK

\subsection{6/thoraxjnl-2014-206260.306}

Introduction Pleural disease is a common health problem in the general population and the number of pleural interventions available to physicians is rapidly expanding. Most clinical studies to date have focused on the generation of successful treatments for pleural diseases without considering patient-centred assessments of symptomatic relief in a procedure undertaken for patient benefit. Patient-related outcome measures (PROMs) such as the assessment of pain and difference in dyspnoea have been used in other disease areas to estimate effectiveness and guide interventions. This prospective study measured PROMs after pleural interventions using a specific survey questionnaire.

Methods Data were collected from 95 patients treated in a tertiary referral centre from December 2013 to June 2014. Pleural interventions included diagnostic aspiration, therapeutic aspiration, thoracoscopy, intercostal chest drain insertion and indwelling pleural catheter insertion. We gathered information on pain, dyspnoea, expected improvement and willingness to repeat the procedure if needed using a $100 \mathrm{~mm}$ visual analogue scale (VAS). Clinical, radiological and histological data were recorded and categorised the patients with pleural effusions to either: malignant, infected, heart failure and undiagnosed. Patients with pneumothorax were classified to either primary or secondary. Data are presented as mean \pm SD.

Results Data were collected from 31 therapeutic aspirations, 30 intercostal drain insertions, 17 diagnostic/simple aspirations, 14 thoracoscopies and 3 indwelling catheter insertions. The results showed the procedure associated with the most pain is medical thoracoscopy (VAS: $20 \pm 20.3 \mathrm{mms}$ ) whereas diagnostic aspiration (VAS: $2.52 \pm 4.78 \mathrm{mms}$ ) was the least uncomfortable. Pain measurements were similar in intercostal and indwelling pleural catheter insertion groups (p: 0.75). VAS score for dyspnoea demonstrated that intercostal drain insertion had the greatest effect on patients' breathlessness compared to the other procedures (VAS difference pre and post-procedure: $50.8 \pm 27 \mathrm{mms}$ ).99.8\% of the patients would repeat any of the pleural procedures if needed.

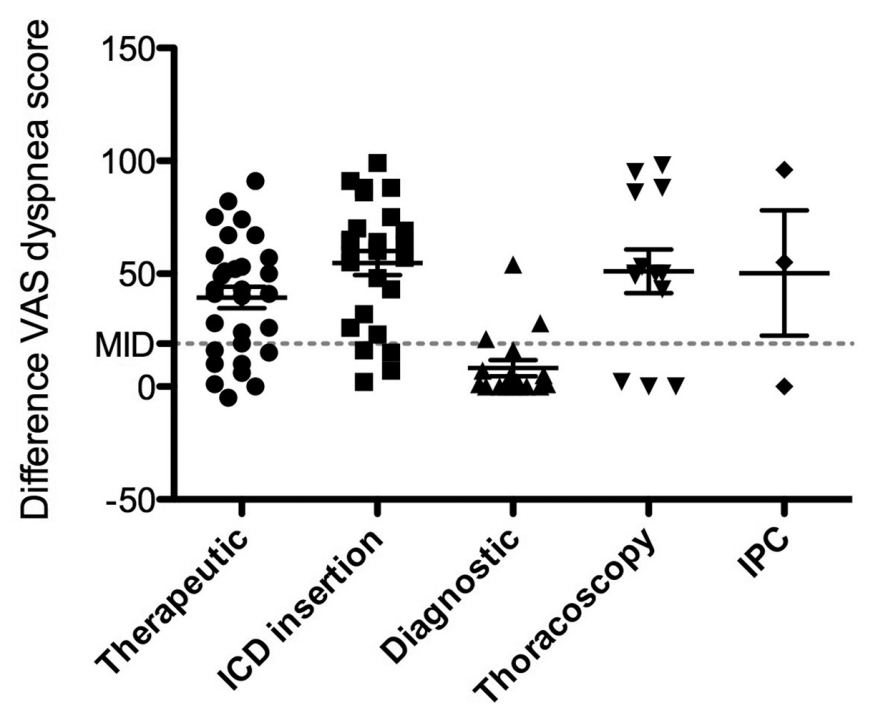

Abstract P177 Figure 1
Conclusion Our study, the first to prospectively assess patientrelated outcomes in pleural procedures, demonstrates that different pleural procedures significantly improve symptoms alongside a high degree of patient satisfaction. Pleural PROMs may represent a standardised way of measuring symptomatic benefit which can be used in both clinical practice and future research.

\section{P178 CLINICIAN AND PATIENT EXPERIENCE IN THE DELIVERY OF A DAY-CASE LOCAL ANAESTHETIC THORACOSCOPY SERVICE AT A SPECIALIST PLEURAL UNIT}

I Psallidas, JP Corcoran, RJ Hallifax, A Talwar, A Sykes, NM Rahman. Oxford Centre for Respiratory Medicine, Oxford University Hospitals NHS Trust, Oxford, UK

\subsection{6/thoraxjnl-2014-206260.307}

Background and method Local anaesthetic thoracoscopy (LAT) is utilised by an increasing number of respiratory physicians for diagnostic and therapeutic purposes in the setting of pleural disease. Although guidelines [1] allow for day-case LAT (i.e. procedure and discharge home on the same day), the majority of UK centres electively admit patients for overnight observation postprocedure. This impacts on service provision by increasing bed occupancy and limiting procedural capacity; whilst affecting patients by incurring a hospital stay they might not need. Reasons for centres not offering day-case LAT are unclear but may include clinician experience and limitations in the available guidelines.

Day-case LAT has been offered by our tertiary centre-based pleural service for a number of years. Patients are routinely considered for this approach if their procedure is for purely diagnostic purposes, as opposed to being therapeutic in addition (i.e. whether talc poudrage pleurodesis is anticipated). Other factors considered in the decision-making process include performance status, co-morbidities and social background. A review of our procedural database from January 2010 to June 2014 was

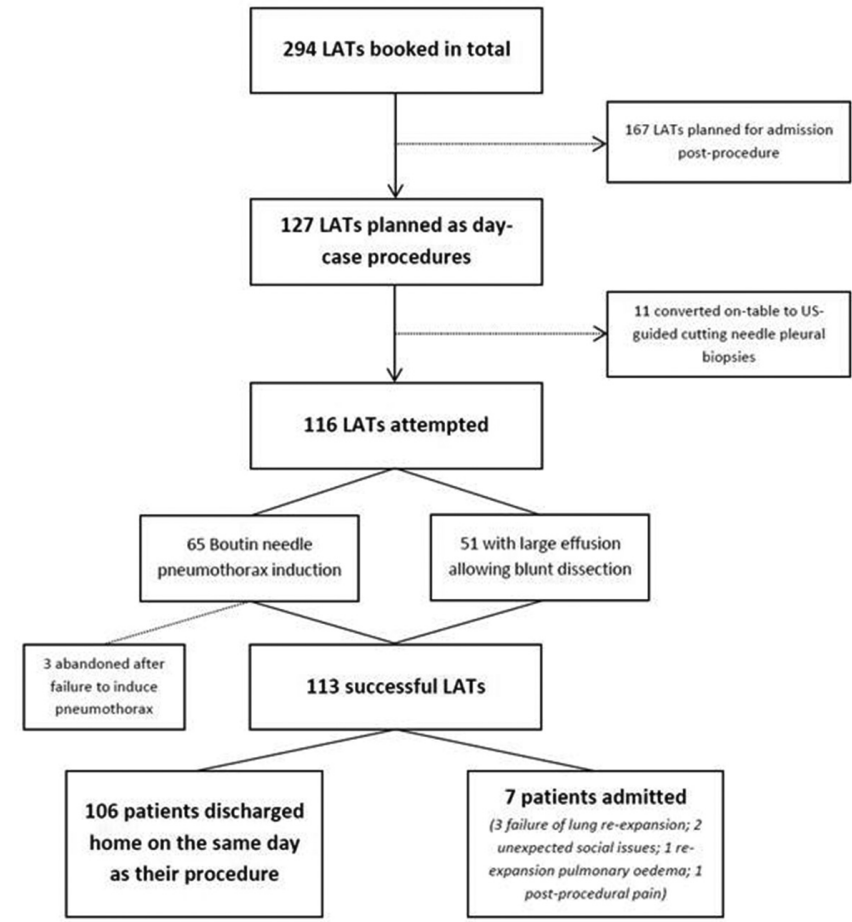

Abstract P178 Figure 1 Flowchart of day-case LAT selection and outcomes (January 2010-June 2014) 
performed to identify the number of day-case LATs and define the characteristics of the patient population offered this approach.

Results Of 294 LATs booked during this period, 127 (43.2\%) were planned as a day-case procedure. 113 day-case LATs went ahead with 7 patients (6.2\%) requiring an "unplanned" hospital admission for reasons outlined in Figure 1. Patients planned for day-case LAT tended to be younger (68.1 vs. 72.4 years, $\mathrm{p}=$ 0.12 , unpaired t-test) with fewer co-morbidities and better social support than the general population having this procedure. No patient declined a day-case procedure having been offered one, whilst the process on the day proved acceptable with most patients valuing the opportunity to avoid an overnight hospital stay.

Conclusion With careful case selection day-case LAT can be provided successfully, benefiting patients and clinicians whilst saving bed days and healthcare costs. Centres with the appropriate case mix and experience may wish to develop day-case LAT as part of their service. Future guidelines should acknowledge this need and offer advice on patient selection and logistical requirements.

\section{REFERENCE}

1 Thorax 2010; 65 Suppl 2:ii54-60

\section{P179 DOES DAY-CASE THORACOSCOPY REDUCE THE NUMBER OF HOSPITAL BED DAYS FOR PATIENTS UNDER INVESTIGATION FOR UNILATERAL PLEURAL EFFUSION?}

J Fallon, J Pepperell. Musgrove Park Hospital, Taunton, UK

\subsection{6/thoraxjnl-2014-206260.308}

Introduction and objectives Medical thoracoscopy is the investigation of choice for diagnosis of exudative pleural effusions where pleural cytology is negative and malignancy is suspected. Over the past 18 months, our centre has offered day-case thoracoscopy to suitable patients, with the aim of reducing elective admissions and overall hospital bed days for patients undergoing this procedure. A review of practice has been performed to evaluate whether day-case thoracoscopy is effective in reducing bed days in this cohort of patients.

Methods Data was retrospectively collected from patients attending our centre for medical thoracoscopy over a 12 month period (Feb 2013 - Feb 2014). Information on the number of bed days required, readmission rates and repeat procedures was reviewed for those attending for day-case thoracoscopy and compared with those electively admitted for overnight stay +/talc pleurodesis.

Results Of 53 patients undergoing medical thoracoscopy at our centre, 31 patients were electively admitted for thoracoscopy, with an average stay of 3.8 bed days. 23 (74\%) of this group underwent talc pleurodesis during their initial admission. 13 (42\%) patients required readmission within 6 months, extending their total length of stay in this period to 7.3 bed days. 11 $(35 \%)$ of these patients required further pleural procedure (s) within 6 months of initial thoracoscopy. 22 patients underwent day-case thoracoscopy (initial stay of 0 bed days). 9 (41\%) of these patients required re-admission within 6 months, with an average total length of stay of 3.8 bed days. 11 (50\%) of these patients went on to require further pleural procedure (s). For the 22 patients attending for day-case thoracoscopy, the number of bed days saved (number of patients $\mathrm{x}$ average length of elective admission) was 83.6 bed days.
Abstract P179 Table 1 Comparison of elective admission for thoracoscopy with day-case thoracoscopy

\begin{tabular}{lll}
\hline Thoracoscopy patients $(\mathbf{n}=\mathbf{5 3})$ & $\begin{array}{l}\text { Elective admissions } \\
(\mathbf{n}=\mathbf{3 1})\end{array}$ & $\begin{array}{l}\text { Day-case } \\
(\mathbf{n}=\mathbf{2 2})\end{array}$ \\
\hline Initial admission bed days (average) & 3.8 & 0 \\
Re-admission within 6 months & $13(42 \%)$ & $9(41 \%)$ \\
Re-admission bed days (average) & 3.5 & 3.8 \\
Total bed days (average) & 7.3 & 3.8 \\
Further pleural procedure (s) within 6 months & $11(35 \%)$ & $11(50 \%)$ \\
\hline
\end{tabular}

Conclusions Day-case thoracoscopy reduces the number of hospital bed days in patients under investigation for unilateral pleural effusion and does not lead to increased rates of re-admission for this cohort of patients. Those undergoing day-case thoracoscopy are more likely to require a further pleural procedure, as talc pleurodesis is not performed in this group.

\section{P180 THE IMPORTANCE OF ACCURATE PRE-OPERATIVE BIOPSY IN THE RADICAL MANAGEMENT OF MESOTHELIOMA - DON'T BLAME THE PHYSICIAN JUST KEEP TAKING MORE BITES}

AJ Sharkey, R Vaja, V Joshi, J Le Quesne, S Muller, A Nakas, C Richards, D Waller. Glenfield Hospital, Leicester, UK

\subsection{6/thoraxjnl-2014-206260.309}

Introduction Histological subtype is an independent prognostic factor in malignant pleural mesothelioma (MPM). Accurate typing is required to offer appropriate therapy, with surgery generally being reserved for epithelioid disease due to the poor survival in biphasic and sarcomatoid MPM. Preoperative tissue can be obtained by a variety of methods which may yield suboptimal specimens.

We aimed to investigate whether the mode of biopsy influenced the accuracy of diagnosis.

Methods We reviewed clinicopathological data from all patients who underwent radical surgery for MPM from 2000-2014, and compared subtyping from biopsies and resection specimens. In addition, a subspecialty expert consultant histopathologist reviewed biopsies from all biphasic cases.

Results In total, 335 patients had available pathological data available.

61(18.2\%) showed discordance in subtyping between the diagnostic biopsy and the resection specimen. In 53 patients a poorer prognosis cell type was identified at resection (see Table).

There was poorer survival in the discordant group; median survival 8.2 vs 15.2 month ( $\mathrm{p}=0.001 \mathrm{HR}=1.659$ 95\% CI 1.227-2.243)

Discordance was found to be an independent predictor of survival on multivariate analysis (HR 1.653 95\% CI 1.207-2.264 $\mathrm{p}=0.002$ ).

There was no effect of method of pre-operative biopsy on concordance $(p=0.306)$. There was also no difference in the accuracy of the diagnosis if a surgical biopsy was performed versus medical thoracoscopy or a radiologically guided biopsy $(\mathrm{p}=0.768)$.

In $26(18.4 \%)$ cases there was discordance between pre-operative biopsy and post-operative histological subtype. In 22 patients a poorer prognosis cell type was identified.

Conclusion These data demonstrate potential inaccuracy of current biopsy practice with accompanying impaired patient 